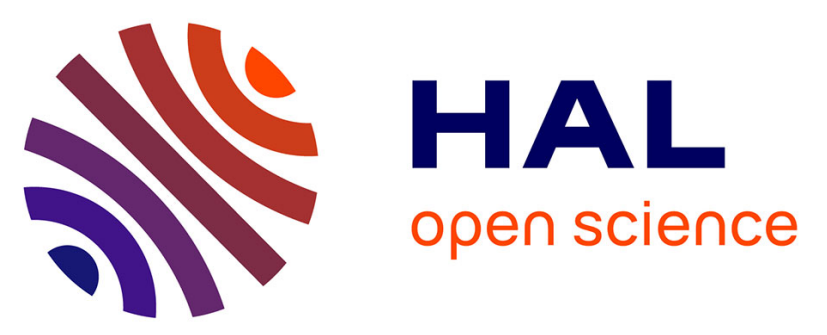

\title{
Adherence and effectiveness of drug combination in curative treatment among children suffering uncomplicated malaria in rural Senegal.
}

Aurélia Souares, Richard Lalou, Ibra Sene, Diarietou Sow, Jean-Yves Le Hesran

\section{To cite this version:}

Aurélia Souares, Richard Lalou, Ibra Sene, Diarietou Sow, Jean-Yves Le Hesran. Adherence and effectiveness of drug combination in curative treatment among children suffering uncomplicated malaria in rural Senegal.. Transactions of The Royal Society of Tropical Medicine and Hygiene, 2008, 102 (8), pp.751-8. 10.1016/j.trstmh.2008.05.016 . ird-00418639

\section{HAL Id: ird-00418639 \\ https://hal.ird.fr/ird-00418639}

Submitted on 21 Sep 2009

HAL is a multi-disciplinary open access archive for the deposit and dissemination of scientific research documents, whether they are published or not. The documents may come from teaching and research institutions in France or abroad, or from public or private research centers.
L'archive ouverte pluridisciplinaire HAL, est destinée au dépôt et à la diffusion de documents scientifiques de niveau recherche, publiés ou non, émanant des établissements d'enseignement et de recherche français ou étrangers, des laboratoires publics ou privés. 


\title{
Adherence and effectiveness of drug combination in curative treatment among children suffering uncomplicated malaria in rural Senegal
}

\author{
Aurélia Souares ${ }^{a, *}$, Richard Lalou $^{\mathrm{b}}$, Ibra Sene ${ }^{\mathrm{c}}$, \\ Diarietou Sow ${ }^{d}$, Jean-Yves Le Hesran ${ }^{a}$
}

\author{
a Institut de Recherche pour le Développement, UR10, Faculté de pharmacie, 4 av de l'Observatoire, 75006 Paris, France \\ b Institut de Recherche pour le Développement, UMR151-LPED, Centre St Charles, Case 10, 13331 Marseille cedex 3, France \\ c District sanitaire de Mbour, Mbour, Senegal \\ 'District sanitaire de Thies, Thies, Senegal
}

Received 12 October 2007; received in revised form 22 May 2008; accepted 22 May 2008

\section{KEYWORDS \\ Malaria; \\ Drug combination \\ therapy; \\ Adherence; \\ Treatment outcome; \\ Child; \\ Senegal}

\begin{abstract}
Summary Increased Plasmodium falciparum resistance to chloroquine has prompted national malaria programs to develop new policies in several African countries. Less than a year after the introduction of amodiaquine/sulfadoxine-pyrimethamine (AQ/SP) as first-line treatment in Senegal, we examined adherence rates to therapy and its efficacy among children. The study was conducted in five dispensaries in rural Senegal. Children aged $2-10$ years with a presumptive diagnosis of malaria were prescribed AQ/SP. Thick blood film analyses were carried out on days $0,3,7,14$ and 28 . Blood and urine samples were collected on day 3 for drug level measurements. The principal caregivers were questioned on treatment adherence. Among the 289 recruited children, 144 had a parasitemia $>2500 / \mu$ l. The results demonstrated markedly good efficacy for the treatment, as no detectable parasitemia was observed on day 28 for $97.9 \%$ of the children. However, we noticed that $35.3 \%$ of children did not comply with the recommended doses and $62.3 \%$ did not exactly adhere to the drug schedule. Despite the good efficacy of the drugs, adherence to the therapeutic scheme was poor. Strategies to promote patient adherence would improve drug performance and thus might help to prevent the rapid emergence of drug resistance.

(c) 2008 Royal Society of Tropical Medicine and Hygiene. Published by Elsevier Ltd. All rights reserved.
\end{abstract}

\section{Introduction}

Each year, malaria is responsible for 300 to 500 million new cases worldwide and accounts for 1 to 2 million deaths, predominantly among young children in Africa. For decades, chloroquine (CQ) was used as first-line treatment. High drug
* Corresponding author. Tel.: +33 1 53731507; fax: +33153739617. E-mail address: souares@uni-heidelberg.de (A. Souares). 
pressure through self-medication, poor treatment adherence and under-utilization of health facilities has caused CQ resistance in many sub-Saharan countries (Nuwaha, 2001). This resistance led to an increase in mortality and morbidity and prompted national malaria programs to develop new policies in most African countries. Combination therapy with antimalarial drugs that have different modes of action has been shown to improve the treatment efficacy of individual drugs (WHO, 2001) and decrease the risk of resistance. The introduction of artemisinin-based combination therapy (ACT) has been shown to increase cure rates (White, 1999) and has been officially recommended by WHO (2001).

Nearly all high-burden African countries have seen an unprecedented change in national drug policies in recent years. In line with the WHO recommendations, they gave up $\mathrm{CQ}$, which had become ineffective, to promote ACT as first-line treatment for mild malaria. If this implementation is official in most of the countries, there are still a large proportion of countries in which the combinations are not available in health facilities and children are treated with less effective monotherapies. Data available between 2004 and 2006 in 14 countries supposedly already using ACT showed that in reality it has been used for less than $6 \%$ of febrile children (UNICEF, 2007). Moreover, there is little evidence concerning adherence to ACT and other therapeutic combinations to date. The new therapy may be subject to the same pressures and might suffer a setback similar to that of CQ (White, 2004).

Before a broad utilization of ACT becomes successful throughout the African continent, several issues need to be addressed (Bloland et al., 2000), particularly that of patient treatment adherence: the degree to which a patient adheres to the prescribed treatment schedule. Poor patient adherence has serious consequences in terms of drug effectiveness. It is likely to be lower for patients who do not complete their treatment (Yeung and White, 2005).

Faced with an average CQ treatment failure of 25\% (Gaye, 2002), Senegal changed its national malaria treatment policy from CQ to amodiaquine/sulfadoxine-pyrimethamine (AQ/SP) in 2003. In 2006, they shifted to ACT. The introduction of this combination therapy can be considered a test before the implementation of ACT in terms of health staff behavior and public adherence to new drugs and drug combinations.

Less than a year after the introduction of $A Q / S P$ as firstline treatment in Senegal, this study was undertaken to examine patient adherence rates and drug effectiveness among children.

\section{Materials and methods}

\subsection{Study area and population}

The study was conducted in the rural areas of Mbour and Thies: two departments situated about $90 \mathrm{~km}$ southeast of Dakar, capital city of Senegal. The population in this region is mainly Sereer. Millet and groundnuts are the principal traditional staple crop and cash crop, respectively. The farmers in this area also raise cattle. To cope with the agricultural crisis, new activities have arisen, such as temporary migration to urban centers, particularly to Mbour and tourist areas.
Malaria is endemic in this area, with seasonal outbreaks. The annual rainy season is short (July-October). Malaria transmission is mainly restricted to the months from August to November. The study took place in 2004 during the rainy season. We chose five health centers that are representative of health-care provision in rural Senegal (staff and technical means, population scattering around the health centers, recruitment area). Urban and tourist areas and health centers with special non-governmental organization health interventions were avoided. A homogeneous geographic area representing 30037 people was defined.

The selected health centers provide basic services to the study population: curative care, immunization, pre-natal care, delivery and malnutrition management. The health structures are staffed by nurses and birth attendants. There are no permanent physicians, midwives, laboratory facilities or emergency transportation. Health centers were selected in terms of patient attendance in order to obtain the daily minimum patient recruitment among children.

\subsection{Design and procedures}

During the study period, nurses identified all the children, aged between 2 and 10 years, who presented with fever (axillary temperature $>37.5^{\circ} \mathrm{C}$ ) and a presumptive diagnosis of mild malaria during an initial consultation. A thick blood film was done to measure the parasitemia. A urine sample was collected to evaluate $C Q$ intake before the consultation.

Since the adoption of the new policy, all children are prescribed a single dose of SP (25 mg sulfadoxine/kg; $1.25 \mathrm{mg}$ pyrimethamine $/ \mathrm{kg}$ ) on day 0 (D0), and one daily dose of AQ for $3 \mathrm{~d}(10 \mathrm{mg} / \mathrm{kg} / \mathrm{d})$ (Table 1). Each provider was given a chart showing the appropriate dosage by weight and age, defined by the Senegalese National Malaria Control Program. The program did not expect nurses to supervise the first intake. Drugs were available at the health center pharmacy. No study team member was present at the health center. We received the identification form from the nurse and recruited the children the day after the last treatment dose day 3 (D3), when we visited mothers/guardians at home to explain the study and collect their written consent. Apart from the study team, no one was aware of this visit before our arrival. A questionnaire was administered at D3 to caregivers and adults who accompanied the children to the health center, with questions related to treatment adherence, history of the febrile illness, knowledge and source of information about antimalarial drugs and awareness of the recent policy change.

\subsection{Effectiveness}

For the effectiveness study, thick blood samples were carried out at $\mathrm{D} 0,3,7,14$ and 28 . In the absence of laboratories in the health centers, samples were read in the laboratory of the French Institute of Research for Development in Mbour. Children with Plasmodium falciparum monoinfection and parasitemia $>2500$ parasites $/ \mu$ l at D0 were diagnosed positively with malaria. Therapeutic outcome was classified into early treatment failure, late clinical failure, late parasitological failure or adequate clinical and parasitological response (WHO, 2003). Parasites were counted using 
Table 1 Age-specific dosing schedule for sulfadoxine-pyrimethamine (SP) plus amodiaquine (AQ) in Senegal

\begin{tabular}{|c|c|c|c|c|c|}
\hline \multirow[t]{2}{*}{ Age group } & \multirow[t]{2}{*}{ Weight (kg) } & \multirow{2}{*}{$\begin{array}{l}\text { SP tablets }{ }^{\mathrm{a}} \\
\text { D0 }\end{array}$} & \multicolumn{3}{|l|}{ AQ tablets ${ }^{\mathrm{b}}$} \\
\hline & & & DO & D1 & D2 \\
\hline $2-11$ months & $5-10$ & 0.5 & 0.5 or 2 tsp & 0.5 or 2 tsp & 0.5 or 2 tsp \\
\hline $1-2$ years & $10.1-14$ & 0.75 & 0.75 or $3 \mathrm{tsp}$ & 0.75 or 3 tsp & 0.75 or 3 tsp \\
\hline $3-5$ years & $14.1-20$ & 1 & 1 or 4 tsp & 1 or 4 tsp & 1 or 4 tsp \\
\hline $6-8$ years & $20.1-30$ & 1.5 & 1.5 & 1.5 & 1.5 \\
\hline $9-11$ years & $30.1-40$ & 2 & 2 & 2 & 2 \\
\hline $12-13$ years & $40.1-50$ & 2.5 & 2.5 & 2.5 & 2.5 \\
\hline$>14$ years & $>50$ & 3 & 3 & 3 & 3 \\
\hline
\end{tabular}

Giemsa-stained thick blood films and parasite density was calculated based on an assumed mean white blood cell count of $8000 / \mu \mathrm{l}$. All thick blood films were read twice. A slide was declared negative after 200 negative microscopic fields. The pre-treatment of CQ was assessed with urine samples at D0 with the Haskins and Mount, or HMM II, method using methyl orange (Mount et al., 1989).

\subsection{Definition of adherence}

To measure adherence, we compared the caregivers' administration of the drugs with the nurses' prescription. We defined two indicators of adherence. Firstly, we considered those who took $80 \%$ of the prescribed dose of the two drugs to be adherent (Dahan et al., 1985). We totaled the entire drug intake declared and compared the total dose with the total dose prescribed by the nurse. Secondly, strict full adherence (SFA) described a patient as fully adherent to the prescription, including dose, duration and frequency. Patients were required to take the exact daily dose of $A Q$ prescribed for $3 \mathrm{~d}$ and the exact dose of SP prescribed on the first day. In the case of syrup, the drug providers gave caregivers the instructions to take 2, 3 or 4 teaspoonfuls a day. We considered a teaspoonful to hold $5 \mathrm{ml}$.

\subsection{Data analysis}

Questionnaire data were entered in Dbase and analyzed using Stata 8 (Stata Corp., College Station, TX, USA). Sample characteristics and patient classification were described as proportions. Free and informed consent was sought from the children's caregivers before administration of the questionnaires and blood samples.

\section{Results}

\subsection{Population}

Of the 304 patients aged $2-10$ years considered by the nurse to have mild malaria, 289 were included in the study. The remaining 15 were prescribed quinine by the nurse. One hundred and forty-four children had laboratory-confirmed malaria (parasitemia $>2500 / \mu \mathrm{l}$ ) and 145 were diagnosed presumptively by the nurse (117 were negative and 28 had a parasitemia $\leq 2500 / \mu \mathrm{l})$. The median age of the recruited children was 5.4 years. The sex ratio (male/female) was 1.3. The mother was the caregiver in $69.5 \%$ of cases, followed by the grandmother (13.2\%), the father (7.6\%) and others (9.7\%). Over $66 \%$ of the children had a history of fever in the previous $24 \mathrm{~h}$, and $54.3 \%$ had an axillary temperature over $37.5^{\circ} \mathrm{C}$ at the time of consultation (Table 2). The urine test results showed that $6 \%$ of the children took CQ before the consultation at the health center.

To compare compliance data from the questionnaire with blood drug level, we collected blood samples at D3 from children who had a parasite density $>2500 / \mu$ l at D0. Sulfadoxine and pyrimethamine concentrations were determined in whole blood using the SPE-HPLC method from the blood sample taken at D3 (Pussard et al., 1986).

All children were prescribed either SP and AQ (70.6\%) tablets or syrup (29.4\%). Nearly all $(92.1 \%)$ were prescribed an antipyretic (80.7\% aspirin and $11.4 \%$ paracetamol); $27 \%$ were prescribed a fourth drug; and $4.8 \%$ were prescribed a fifth drug, usually an antibiotic or antiparasite medication.

Fourteen caregivers $(5.9 \%)$ did not buy all the drugs prescribed: two of them $(0.7 \%)$ did not buy $A Q$; six $(2.1 \%)$ did not buy SP; eight (2.8\%) did not buy an antipyretic; and six (2.1\%) did not buy another drug.

The mean cost of the prescription is approximately 577 CFA (225-1650) (€0.88 [0.34-2.52]) and is more expensive for young children, aged $2-4$ years $(711 \mathrm{CFA}=€ 1.08)$, than for older children, aged $8-10$ years $(453$ CFA $=€ 0.69)$, because of the price of the syrup.

\subsection{Adherence}

Treatment adherence was measured for all 289 children. Four $(1.4 \%)$ caregivers said they did not give $A Q$, and 17 said they did not give SP (5.9\%). Seventeen children (5.9\%) did not take one of the two drugs. Four (1.4\%) took neither $A Q$ nor SP.

\subsubsection{Daily dosage}

The daily dosage administered for AQ varied: $60.2 \%$ administered the correct dosage each day; $12.8 \%$ overdosed it; $2.8 \%$ underdosed it; and 24.2\% administered different dosages (both under- and overdosing) over the $3 \mathrm{~d}$ period. For SP on 
Table 2 Main characteristics of the study subjects

\begin{tabular}{lcc}
\hline & $\begin{array}{c}\text { Nurses' clinical diagnosis of malaria } \\
\text { with positive blood film }\end{array}$ & $\begin{array}{c}\text { Nurses' clinical diagnosis of malaria } \\
\text { with negative blood film }\end{array}$ \\
\hline No. individuals & 144 & 145 \\
No. females (\%) & $61(42.3)$ & $64(44.1)$ \\
Mother as caretaker (\%) & $102(70.8)$ & $99(68.2)$ \\
Caretaker with no formal education & $131(91)$ & $109(75.2)$ \\
No. children of the caregiver (mean) & 4.7 & 5.1 \\
Median age (year, range) & $5.5(1-10)$ & $5.3(2-12)$ \\
Median weight (kg, range) & $16.5(7-30)$ & $16.1(8-38)$ \\
Mean temperature ( ${ }^{\circ} \mathrm{C}$, range) & $38.2(36.5-40.9)$ & $38(36.3-40.5)$ \\
Geometric parasitic density $(/ \mu l$, range) & $963(2547-1045333)$ & $637(14-1546)$ \\
Reported or observed symptoms & & $78(53.8)$ \\
Axillary temperature $>37.5^{\circ} \mathrm{C}(\%)$ & $79(54.9)$ & $109(75.2)$ \\
History of fever $(\%)$ & $83(57.6)$ & $58(40)$ \\
Headache $(\%)$ & $45(31.2)$ & $23(15.9)$ \\
Cough (\%) & $13(9)$ & $14(9.6)$ \\
Abdominal pain $(\%)$ & $13(9)$ & $16(12.1)$ \\
Vomiting (\%) & $9(6.6)$ & $9(6.2)$ \\
Diarrhea (\%) & $1(0.7)$ & $3(2.1)$ \\
Anemia (\%) & $7(4.8)$ & \\
\hline
\end{tabular}

the first day, $65.4 \%$ took the correct dose, while $14.2 \%$ took less and $16.9 \%$ took more than was prescribed (Figure 1).

\subsubsection{Treatment duration}

A total of $63.5 \%$ of caregivers administered AQ for exactly $3 \mathrm{~d}$ and $15.1 \%$ continued to give it after the third day. Slightly more than $21 \%$ did not follow the prescribed regimen of three doses of $\mathrm{AQ}$ and omitted one $(17.9 \%)$ or two $(3.5 \%)$ doses. At D2, 16.3\% did not administer AQ. Approximately $12 \%$ did not administer SP as one dose and instead divided it over 2 or $3 \mathrm{~d}$. Just over three percent (3.3\%) of caregivers said they had given SP after the third day and $2.8 \%$ of the caregivers did not administer it on the first day but did on the other days (Figure 2).

\subsubsection{Frequency of daily administration}

Although all caregivers were supposed to give the medication once a day, there was variation in the frequency of administration. Approximately $63.7 \%$ administered it once

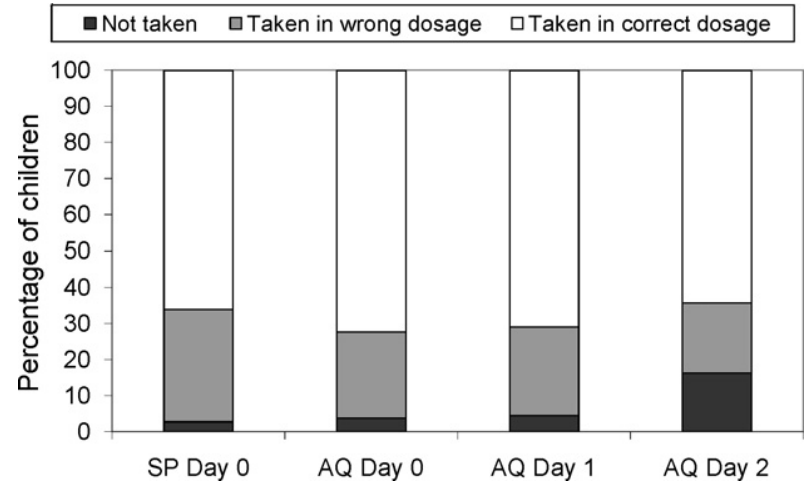

Figure 1 Adherence detailed for each intake. AQ: amodiaquine; SP: sulfadoxine-pyrimethamine. a day, about $6.2 \%$ administered it twice a day $(15.3 \%$ for syrup); $0.7 \%$ administered it three times a day; and $29.4 \%$ administered it a varied number of times over the $3 \mathrm{~d}$ period. A small percentage (1.5\%) administered SP two or three times a day.

\subsubsection{Total dose administered}

Nearly $62 \%$ of children took the exact prescribed dose of AQ (66.5\% for tablets and $52.9 \%$ for syrup); $28.7 \%$ received a lower dose than prescribed $27.5 \%$ for tablets and $32.9 \%$ for syrup); and $9.7 \%$ received a higher dose $(6 \%$ for tablets and $14.2 \%$ for syrup). For SP, $67.5 \%$ took the correct dose, while 9.3\% had underdosed and $23.2 \%$ had overdosed.

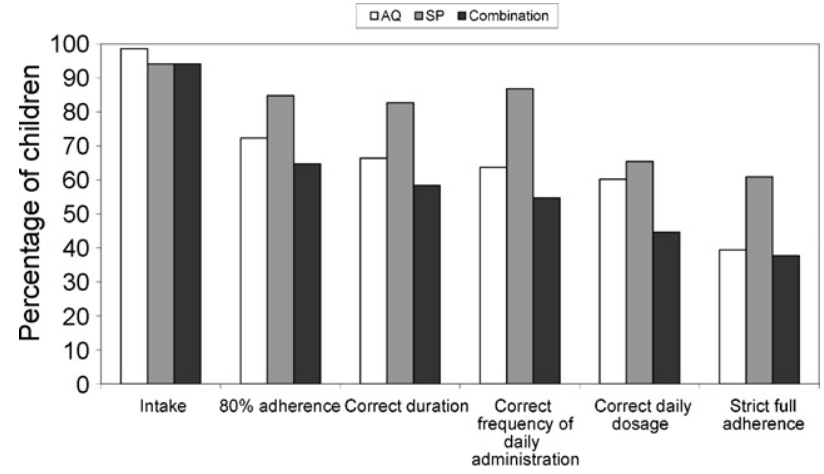

Figure 2 Adherence to the combination amodiaquine/ sulfadoxine-pyrimethamine ( $\mathrm{AQ} / \mathrm{SP})$. Intake: administration of the drug at least one time; $80 \%$ adherence: administration of $80 \%$ of the prescribed dose; correct duration: administration of the drugs $1 \mathrm{~d}$ for SP and $3 \mathrm{~d}$ for AQ; correct frequency of daily administration: administration of one dose a day; correct daily dosage: administration of the correct dosage for each intake; strict full adherence: administration exactly as prescribed by the nurse (dose, duration, frequency). 


\subsubsection{Treatment adherence measurement: $80 \%$} adherent and SFA

Numerous studies examining acute episodes of the disease considered patients to be adherent if they took $80 \%$ of the prescribed dose. Accordingly, 209 (72.3\%) were adherent to AQ and $245(84.8 \%)$ to SP. Hence, a total of 187 caregivers $(64.7 \%)$ were adherent to both drugs. However, only $39.4 \%$ of caregivers demonstrated SFA for AQ and $60.9 \%$ did so for SP. Adherence to treatment with tablets was better than with syrup ( $45 \%$ compared with $28.2 \%$, respectively). Overall, $37.7 \%$ of the caregivers strictly followed the prescription for the two drugs (Figure 2).

\subsection{Biological measure and declarative measure}

Only the children who had a positive parasitemia were included in this part of the study. We considered qualitative results - absence or presence - for HPLC. There is good similarity $(90 \%)$ between interview and HPLC. When comparing interviews with blood concentration, seven (5\%) caregivers said they had given SP to their children and HPLC dosage was negative; and seven (5\%) said they had not given SP and the children were positive.

\subsection{Effectiveness}

All the children with positive parasitemia (144/289) received clinical and parasitological follow-up for $7 \mathrm{~d}$ and $97.9 \%$ $(141 / 144)$ received the follow-up for $28 \mathrm{~d}$. The effectiveness of the treatment was markedly good. No detectable parasitemia was observed at D3 for $87.6 \%$ of the children. Among those who were positive, parasitemia rates were low $(480 / \mu \mathrm{l})$, except for two subjects who had high rates $(>2500 / \mu \mathrm{l})$. We observed one early therapeutic failure; one child remained positive during the entire follow-up but without any clinical signs. This child was classified as non-observant, as his parents bought the drugs but they did not administer it because he felt better. At D28, $97.9 \%$ of the children had a negative thick blood film. We observed two late therapeutic failures: two children became positive again at D28 (Figure 3).

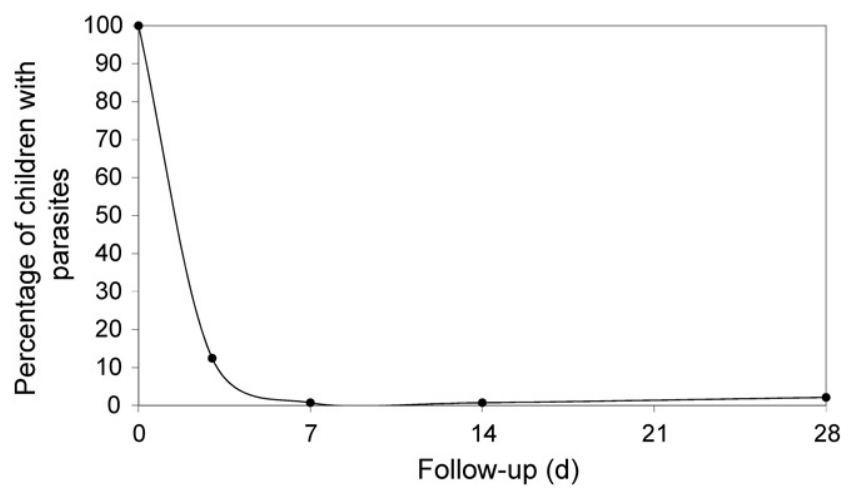

Figure 3 Effectiveness of combination therapy. Effectiveness was measured for all laboratory-confirmed malaria cases $(n=144)$.

\subsection{Drug perceptions and instructions concerning the medication}

All interviewed caregivers and accompanying adults stated that they were not aware of malaria treatment policy changes. Families were unaware of the concept of association: the use of two drugs to treat one disease. AQ was linked to CQ due to its bitterness and the shape of the tablets. SP was not associated with malaria treatment; the caregivers mistook it for an antipyretic because of its similarity in color (white) and size.

The treatment was perceived as difficult to administer to the children by $10.4 \%$ of the caregivers, as children cried (29.1\%) or refused to take the tablets. Slightly over $26 \%$ of the children vomited when the drug was administered or itched after taking the medication. Symptoms played an important role in treatment adherence. For example, $20.1 \%$ thought it was unnecessary to continue the treatment if their child no longer appeared sick. The majority (90.3\%) of the caregivers said that they were not provided with information concerning the prescribed drugs at the time of consultation. Most of them (72.3\%) had been provided with information by the pharmacist; however, $68 \%$ of them reported that the information on drug intake was inadequate.

\section{Discussion}

High drug pressure through self-medication, poor adherence and under-dosage has led to $\mathrm{CQ}$ resistance and frequent therapeutic failures (Basco, 2004; McCombie, 1996). In 2004, Senegal changed its antimalarial strategy for AQ/SP in order to tackle this resistance. Our study aimed to analyze population adherence to the new strategies for treating malaria.

\subsection{Treatment adherence to the $A Q / S P$ combination}

Few studies are available on antimalarial combination therapy adherence, and the lack of a uniform methodology makes true comparison difficult (Yeung and White, 2005). A wide range of definitions concerning adherence are typically used. Additionally, only a small proportion of the studies used detailed definitions of adherence that included doses, duration and frequency (Ansah et al., 2001; Depoortere et al., 2004; Yepez et al., 2000). This detailed definition is more specific but also more complex and requires reconstruction of the entire drug-intake history. For this study we used two treatment adherence indicators. First, we used a definition of $80 \%$ adherence, signifying that caregivers had given more than $80 \%$ of the prescribed dose to their children (Dahan et al., 1985). We also used a very precise definition, called SFA. Only those patients who took all the prescribed medication for the correct number of days and at the correct dosage each day were classified as strictly adherent.

We compared our results from both indicators to results from other studies. If we considered only the intake quantity, adherence at the $80 \%$ level was weaker in our study $(64.7 \%)$ than that described by Fogg et al. (2004) in Uganda 
(91\%), where a new drug combination was tested, or by Ansah et al. (2001) in Ghana (90\%), where new CQ packaging was introduced. Our results were more closely related to the Reilley study in Sri Lanka, where $74 \%$ of patients were adherent when only total medication intake was considered (Reilley et al., 2002). The large differences might be explained by particular study conditions; for example, new drugs or new packaging tests (Ansah et al., 2001; Fogg et al., 2004). In these studies, the use of written consent at D0 and caregiver training induced differences in the usual health practices and may have had an impact on patient behavior, as people were aware of the research before treatment had commenced (Osterberg and Blaschke, 2005). We tried to measure adherence while limiting similar biases as much as possible. We did not modify any of the health center's operations; no team member was present at the health center, and patients knew about the study only once the treatment had supposedly finished.

Only $37.7 \%$ of patients were adherent in this study if we used the definition of SFA. Depoortere et al. (2004), using the same indicator, found that $39 \%$ of patients were compliant to a $3 \mathrm{~d}$ regimen of AS/SP. Adherence concerns not only medication intake but also treatment duration, dosage and frequency of administration. The SFA permits us to take into account all of these components.

\subsection{Measures of adherence}

Some previous studies have shown that caregivers or selfreported drug histories were subject to biases and errors and are thus not reliable (Nwanyanwu et al., 1996) and would need to be validated by drug concentration (Yeung and White, 2005). Our data collection required a long and detailed caregiver interview and allowed us to obtain reliable data for biological assessment. Blood drug assays were carried out in our study and we obtained good agreement between the questionnaire and blood concentration of the drugs. However, it was not possible to highlight the link between dose intake and blood drug level for each compound. Blood drug concentrations cannot be translated into a strong estimate of adherence, because levels vary considerably between individuals, depending on a variety of uptake, metabolic and elimination factors (Souares, unpublished data). The same conclusion was reported in Uganda with artemether-lumefantrine (Fogg et al., 2004). The differences between stated behavior and blood sample have been investigated and might be explained by confusion between SP and antipyretics; SP tablets were mistaken for antipyretics because of their shape (they are a little bigger) and their white color. Caregivers may have confused them and did not realize that they took two different drugs. Other authors also showed that the reported behavior has an advantage over blood levels in determining how the drug was administered (Kofoed et al., 2003; Qingjun et al., 1998). Asking patients non-judgmentally about medication-taking behaviors and decreasing the delay between treatment and questionnaire administration (D3, in our survey) are practical strategies for identifying poor adherence and reducing possible error or distorted information (Osterberg and Blaschke, 2005).

\subsection{Relation between adherence and effectiveness}

Treatment adherence and effectiveness are closely related; a patient cannot be cured without taking their drugs and "drugs don't work in patients who don't take them" (Osterberg and Blaschke, 2005). In our study, despite low adherence among $35.3 \%$ of patients, we found a high rate of efficacy: $97.9 \%$ of the children had a negative parasitemia at D28.

Sokhna et al. (2001) found that, at D14, the percentages of in vivo resistant infection to AQ or SP were 16 and $7 \%$, respectively, for 266 Senegalese children with uncomplicated malaria. Thus, this strongly suggests that each drug is effective on its own. If caregivers gave their child at least one of the two drugs, they were likely to be cured. Moreover, the drug therapy combination reinforces treatment effectiveness. At D28, no therapeutic failure was noted among 181 patients who were administered both $A Q$ and SP (Gaye, 2002). In Burkina Faso, Zongo et al. (2005) found that this treatment was effective $95.8 \%$ of the time, and Mockenhaupt et al. (2005) in Ghana observed an even higher rate of effectiveness (98\%). Similar results were reported from Rwanda and Uganda, with $97.7 \%$ efficacy at D28 (Dorsey et al., 2003; Rwagacondo et al., 2003).

Our results also show that 47.7 and $86.1 \%$ of patients using $A Q$ and $S P$, respectively, received a dose greater than the theoretical dose. In interviews, nurses explained they choose to overdose children by taking age into account instead of weight for underweight children or because of the particular seriousness of the disease. This problem is also linked to the tablets, which are difficult to divide and cannot be adapted to an exact dose corresponding to body weight. As a result, children may have taken the curative dose even if they were non-adherent to the prescription.

However, good drug effectiveness, as we observed in our study, could have a negative impact on treatment adherence. One caregiver out of five omitted at least one dose, more often the last one (16.3\%). This result corroborates the attitudes of the family: they declared they did not give the tablets to the child after the symptoms stopped. In Ecuador, Yepez noticed among others factors that "getting cured quickly' also did not help adherence (Yepez et al., 2000).

\subsection{Barriers to adherence and potential for ACT implementation}

Data collected on adherence to AQ/SP combinations inform us about problems that might be encountered with ACT. However, while AQ/SP were separated, the ACT, at least for the two main combinations artesunate/amodiaquine (AS/AQ) and artemether/lumefantrine (AL), are available in a single blister pack integrating both molecules. This is expected to simplify the drug intake and validate the concept of association in people's minds. But before a coformulation, such as Coarsucam, is available worldwide, the main problem concerns the number of tablets and lack of pediatric form for one of the two principal combinations (AS/AQ). Co-formulation will limit the number of tablets to take and should thereby promote adherence to treatment. However, even if these elements should 
facilitate drug intake, poor adherence to treatment seems to be principally linked to a lack of understanding. In our study, caregivers' interviews revealed the lack of information received at the health center. It was shown that detailed information, notably using pictures and verbal instructions, could improve adherence from 36.5 to $73.3 \%$ in a survey made in Nigeria (Okonkwo et al., 2001). In particular, families need information on the differences between symptoms and the outcome of the disease. Unfortunately, the nurses themselves did not receive much information on the new therapy, which did not help them to inform their patients and patients' caregivers (Souares et al., 2006). For a successful implementation and utilization of ACT in Africa, countries should really focus on nurse training and diffusion of information among the population in relation to the introduction of new drug combinations.

\section{Conclusions}

Efforts must be made to better understand factors that might impact compliance and improve it by helping caregivers to adhere to the prescribed treatment. The provision of packaging such as pre-packet tablets, co-formulated treatment therapy or a container with the required volume and a measuring spoon for syrup could also have an impact on mothers' adherence to recommended doses. Nevertheless, in the absence of pertinent training for health center staff and specific communication to patients and caregivers, new policy implementations were and will continue to be accompanied by misunderstanding and non-adherence. Lessons from this transitional experience in Senegal would be useful for ACT implementation and should focus the attention of policymakers on patient and health staff adherence to treatment. Non-adherence to ACT treatment could result in an increase in the number of therapeutic failures with the subsequent emergence of chemoresistance. In the long term, these different processes could lead to a failure of this new malaria control strategy.

Authors' contributions: AS, RL, DS, IS and JYLH designed the study protocol; AS, RL, DS and IS carried out the study; AS, RL and JYLH analysed and interpreted the data; AS and JYLH drafted the manuscript. All authors revised the article critically and read and approved the final manuscript. AS and JYLH are guarantors of the paper.

Acknowledgements: We thank all the staff who participated in the study. We also thank the children who participated in this study and their parents, as well as the health center team members.

Funding: We acknowledge the financial support of Pal+ (Malaria Program of the French Research Ministry) and IRD (Institut de Recherche pour le Développement) and the study grant of 'Fondation des Treilles'.

\section{Conflicts of interest: None declared.}

Ethical approval: The study protocol was approved by the Senegalese Research Ethics Committee (no. 2052).

\section{References}

Ansah, E.K., Gyapong, J.O., Agyepong, I.A., Evans, D.B., 2001. Improving adherence to malaria treatment for children: the use of pre-packed chloroquine vs. chloroquine syrup. Trop. Med. Int. Health 6, 496-504.

Basco, L.K., 2004. Molecular epidemiology of malaria in Cameroon. XIX. Quality of antimalarial drugs used for self-medication. Am. J. Trop. Med. Hyg. 70, 245-250.

Bloland, P.B., Ettling, M., Meek, S., 2000. Combination therapy for malaria in Africa: hype or hope? Bull. World Health Organ. 78, 1378-1388.

Dahan, R., Dahan, A., Cadranel, J., Caulin, C., 1985. Compliance: measurement of adherence and therapeutic follow-up. Therapie 40, 17-23.

Depoortere, E., Guthmann, J.P., Sipilanyambe, N., Nkandu, E., Fermon, F., Balkan, S., Legros, D., 2004. Adherence to the combination of sulfadoxine-pyrimethamine and artesunate in the Maheba Refugee Settlement, Zambia. Trop. Med. Int. Health 9, 62-67.

Dorsey, G., Vlahos, J., Kamya, M.R., Staedke, S.G., Rosenthal, P.J., 2003. Prevention of increasing rates of treatment failure by combining sulfadoxine-pyrimethamine with artesunate or amodiaquine for the sequential treatment of malaria. J. Infect. Dis. 188, 1231-1238.

Fogg, C., Bajunirwe, F., Piola, P., Biraro, S., Checchi, F., Kiguli, J., Namiiro, P., Musabe, J., Kyomugisha, A., Guthmann, J.P., 2004. Adherence to a six-dose regimen of artemether-lumefantrine for treatment of uncomplicated Plasmodium falciparum malaria in Uganda. Am. J. Trop. Med. Hyg. 5, 525-530.

Gaye, O., 2002. Surveillance de la chimiorésistance du paludisme au Sénégal. Rapport final de la surveillance des résistances dans 8 sites sentinelles. Programme National de Lutte contre le Paludisme. Ministère de l'hygiène, de la santé et de la prévention, Sénégal.

Kofoed, P.E., Lopez, F., Aaby, P., Hedegaard, K., Rombo, L., 2003. Can mothers be trusted to give malaria treatment to their children at home? Acta Trop. 86, 67-70.

McCombie, S.C., 1996. Treatment seeking for malaria: a review of recent research. Soc. Sci. Med. 43, 933-945.

Mockenhaupt, F.P., Ehrhardt, S., Dzisi, S.Y., Teun Bousema, J., Wassilew, N., Schreiber, J., Anemana, S.D., Cramer, J.P., Otchwemah, R.N., Sauerwein, R.W., Eggelte, T.A., Bienzle, U., 2005. A randomized, placebo-controlled, double-blind trial on sulfadoxine-pyrimethamine alone or combined with artesunate or amodiaquine in uncomplicated malaria. Trop. Med. Int. Health $10,512-520$.

Mount, D.L., Nahlen, B.L., Patchen, L.C., Churchill, L.C., 1989. Adaptations of Saker-Solomons test: a simple, reliable colorimetric field assays for chloroquine and its metabolites in urine. Bull. World Health Organ. 67, 295-300.

Nuwaha, F., 2001. The challenge of chloroquine-resistant malaria in sub-Saharan Africa. Health Policy Plan. 16, 1-12.

Nwanyanwu, O.C., Redd, S.C., Ziba, C., Luby, S.P., Mount, D.L., Franco, C., Nyasulu, Y., Chitsulo, L., 1996. Validity of mother's history regarding antimalarial drug use in Malawian children under five years old. Trans. R. Soc. Trop. Med. Hyg. 90, 60-68.

Okonkwo, P.O., Akpala, C.O., Okafor, H.U., Mbah, A.U., Nwaiwu, 0., 2001. Compliance to correct dose of chloroquine in uncomplicated malaria correlates with improvement in the condition of rural Nigerian children. Trans. R. Soc. Trop. Med. Hyg. 95, $320-324$.

Osterberg, L., Blaschke, T., 2005. Adherence to medication. N. Engl. J. Med. 4, 353, 487-497.

Pussard, E., Verdier, F., Blayo, M.C., 1986. Simultaneous determination of chloroquine, amodiaquine and their metabolites in 
human plasma, red blood cells, whole blood and urine by column liquid chromatography. J. Chromatogr. 374, 111-118.

Qingjun, L., Jihui, D., Laiyi, T., Xiangjun, Z., Jun, L., Hay, A., Shires, S., Navaratnam, V., 1998. The effect of drug packaging on patients' compliance with treatment for Plasmodium vivax malaria in China. Bull. World Health Organ. 76 (Suppl. 1), 21-27.

Reilley, B., Abeyasinghe, R., Pakianathar, M.V., 2002. Barriers to prompt and effective treatment of malaria in northern Sri Lanka. Trop. Med. Int. Health 7, 744-749.

Rwagacondo, C.E., Niyitegeka, F., Sarushi, J., Karema, C., Mugisha, V., Dujardin, J.C., Van Overmeir, C., van den Ende, J., D’Alessandro, U., 2003. Efficacy of amodiaquine alone and combined with sulfadoxine-pyrimethamine and of sulfadoxine-pyrimethamine combined with artesunate. Am. J. Trop. Med. Hyg. 68, 743-747.

Sokhna, C.S., Trape, J.F., Robert, V., 2001. Gametocytaemia in Senegalese children with uncomplicated falciparum malaria treated with chloroquine, amodiaquine or sulfadoxine + pyrimethamine. Parasite 8, 243-250.

Souares, A., Lalou, R., Sene, I., Sow, D., Le Hesran, J.Y., 2006. Knowledge and practices among the health workers from the Thies region with regard to new malaria treatment policies. Sante Publique 2, 299-310.
UNICEF, 2007. Malaria and children: progress in intervention coverage. United Nations Children's Fund, New York and Roll back Malaria, Geneva.

White, N.J., 1999. Delaying antimalarial drug resistance with combination chemotherapy. Parassitologia 41, 301-308.

White, N.J., 2004. Antimalarial drug resistance. J. Clin. Invest. 113, 1084-1092.

WHO, 2001. Antimalarial Drug Combination Therapy. Report of a WHO Technical Consultation. World Health Organization, Geneva, WHO/RBM/2001.35.2001.

WHO, 2003. Assessment and Monitoring of Antimalarial Drug Efficacy for the Treatment of Uncomplicated Falciparum Malaria. World Health Organization, Geneva, WHO/HTM/RBM/2003.50.

Yepez, M.C., Zambrano, D., Carrasco, F., Yepez, R.F., 2000. The factors associated with noncompliance with antimalarial treatment in Ecuadorian patients. Rev. Cubana Med. Trop. 52, 81-89.

Yeung, S., White, N.J., 2005. How do patients use antimalarial drugs? A review of the evidence. Trop. Med. Int. Health 10, $121-138$

Zongo, I., Dorsay, G., Rouamba, N., Dokomajilar, C., Lankoande, M., Ouedraogo, J.B., Rosenthal, P.J., 2005. Amodiaquine, sulfadoxine-pyrimethamine, and combination therapy for uncomplicated falciparum malaria: a randomized controlled trial from Burkina Faso. Am. J. Trop. Med. Hyg 73, 826-832. 